International Journal of Modern Physics B, Vol. 6, No. 19 (1992) 3249

(c) World Scientific Publishing Company

\title{
ERRATA
}

\section{MAP COLORING, $q$-DEFORMED SPIN NETWORKS, AND TURAEV-VIRO INVARIANTS FOR 3-MANIFOLDS}

LOUIS H. KAUFFMAN

[Int. J. Mod. Phys. B6, 1765-1794 (1992)]

1. Page 1777 , 4 th line after Section 4 should read:

$$
\text { 1. }\left\langle 2<=A<\check{2}>+A^{-1}<\right) C>
$$

2. Page 1778 , the formula after line 1 should read:

$$
\tilde{\varepsilon}=\left[\begin{array}{cc}
0 & A \\
-A^{-1} & 0
\end{array}\right]
$$

3. Page 1785 , in the last paragraph before Section 6 , the formulae should read:

$$
\left.\square_{0=-2,}\right)_{a}^{a}=+X_{d}^{a}=+\delta_{d}^{a} \delta_{c}^{b}
$$

